\title{
COMPARISON OF THE EFFICIENCY OF MINIMUM-THICKNESS CIRCULAR AND PARABOLIC ARCHES FOR VARIOUS GRAVITY CONDITIONS
}

\author{
Nicos Kalapodis ${ }^{1}$, Georgios Kampas ${ }^{1 *}$, Thomas McLean $^{2}$, Christian Malaga-Chu- \\ quitaype ${ }^{2}$ \\ ${ }^{1}$ University of Greenwich \\ ME4 4TB Chatham, United Kingdom \\ g.kampas@greenwich.ac.uk,n.kalapodis@greenwich.ac.uk \\ ${ }^{2}$ Imperial College London \\ South Kensington Campus London SW7 2AZ, UK \\ thomas.mclean16@imperial.ac.uk, c.malaga@imperial.ac.uk
}

\begin{abstract}
Arches are structural forms that have been used for thousands of years and rely on gravity for their inherent stability. Currently, given the space race for lunar and martian exploration, it has become apparent that soon enough, there will be a need to design and build the first resilient shielding structures on the Moon and Mars against extreme radiation. Arches represent an ideal option for such structures given that they are durable and can be easily built. This paper is based on previous work of the authors and focuses on the stability of circular and parabolic arches with different embrace angles subjected to different levels of equivalent inertial loading in low-gravity conditions. More specifically, it reveals that although parabolic arches can be much more efficient than the corresponding circular for gravitational-only loading, this is not the case for different combinations of inertial loading and embrace angles where the opposite can be true. It highlights the dominant effect of low-gravity conditions on the minimum thickness requirements for both types of arches and considers the effect of a potential additional infill for radiation shielding. Furthermore, this study reveals a self-similar behavior and introduces a "universal" inertial loading.
\end{abstract}

Keywords: Seismic analysis, Low-gravity conditions, Minimum thickness, Failure mechanism, Extraterrestrial structures. 


\section{INTRODUCTION}

Since ancient times, arches and vaulted structures constituted widespread structural forms, due to their inherent advantage to secure a smooth transition of the compressive internal forces from the crown to the springing. Masonry structures, were constructed during the classical period in ancient Greece. Some of those structures were vaults used as entrances to stadiums (Olympia, Nemea). Subsequently, that structural style was further advanced within the Roman period through the construction of bridges, aqueducts and tunnels [1]. In modern history, great engineers had studied the stability of arches beginning with Robert Hooke [2] who was the first to introduce the inverted chain concept with the purpose of describing the path of the compressive resultant force through a catenary (alysoid) shape. Then, Couplet [3] was the first who published his research on the evaluation of the minimum thickness of a semi-circular arch of constant thickness, subjected to its self-weight. However, for the same arch geometry, Milankovitch [4] managed to analytically calculate its minimum thickness and detect the exact locations of the hinges right after the formation of the hinge mechanism. Also, Milankovitch developed an analytical expression for the thrust line and proved that it is not an admissible catenary thrust line. Makris and Alexakis [1] not only verified Milankovitch's results, but also found out that different stereotomies can slightly affect the shape of the thrust line and thus, the minimum required thickness in order for the arch to withstand the applied loading. Additionally, based on Clemente's [5] and Oschendorf's [6] innovative research, Alexakis and Makris [7] extended their research with the evaluation of the minimum thickness of circular arches subjected to both their self-weight and horizontal acceleration that lead to a characteristic fourhinge failure mechanism.

Due to their ability to bridge long spans and their inherent strength, they are considered as suitable structural forms for creating ample space available for the storage of valuable assets or even to provide shelter to future habitable modules in extreme environments. Aiming to create a new space age, various space agencies (NASA, ESA, ISRO, etc) and private firms (SpaceX, Virgin Galactic, Blue Origin, etc) are investing in challenging missions such as "Artemis" amongst others towards the potential human settlement in other planetary bodies starting from the Moon and later on Mars. Thus, there is fertile ground for start conceptualising, designing and then, building the first extra-terrestrial habitats [8] within the coming decades. In their work Kalapodis et al., [9] present a plethora of structural concepts about the design of the prospective extra-terrestrial human habitats. The most predominant such concepts and ideas emphasise the need for an external resilient shielding structure in order to secure valuable assets (energy fuel tanks, robotic elements, future inflatable modules, etc) against harsh conditions like extreme temperature fluctuations[10] and solar radiation [11].

Such extra-terrestrial structures can be made from local soil called regolith [12] in keeping with the In-Situ Resource Utilisation (ISRU) framework [13]. This is proposed to be an archlike structure in order to be used as storage facility and act mainly in compression since regolith, as structural material, is not expected to develop substantial tensile strength [14], while it will be able to provide the necessary compressive strength [15]. Even so, such structures will need to be durable against strong ground motions that, may be generated by shallow moonquakes [16] and marsquakes with epicentres close to the structure or generated by meteoroid impacts [17]. Since the self-weight is reduced due to the reduced gravitational acceleration (although the inertia of the structure remains constant), the structural response will change accordingly $[18,19]$. This research is based on the work of [28] and deals with the limit-state analysis of both circular and parabolic monolithic arches subjected to different levels of horizontal acceleration, for regular gravity and low-gravity conditions, by assuming lower gravitational accelerations, $\alpha \mathrm{g}=\{0.17 ; 0.38\} \mathrm{g}$ in accordance with the lunar and martian gravitational fields 
respectively. Furthermore, a comparison of the efficiency between aches of circular and parabolic geometries is presented.

\section{BACKGROUND OF THE PROBLEM}

Except of the case of semicircular arches, various researchers dealt with the investigation of other arch geometries such as parabolic [20,28], elliptical [21], pointed/gothic [20, 22, 23] and catenary arches $[23,24]$. Most of the works mentioned above, are referred to the static behaviour and stability of the arches through determining their corresponding upper and lower bound thrust lines. According to [25], there are infinite states of equilibrium in an arch; nevertheless, the upper and lower limits for all the associated admissible thrust lines of any arch is indicated by the maximum and minimum thrust transferred to the springing (horizontal component of the reaction force) and are related to a statically determinate three-hinge arch (Fig. 1). [20] Analytically calculated the thrust line of a semicircular arch subjected to both vertical and horizontal loading. Also they proceed with the numerical estimation, through Point Collocation Method [26] and constrained optimisation, of the upper and lower limits of semicircular, parabolic and pointed (statically determinate) arches. Furthermore, $[1,4]$ estimated the minimum thickness of circular arches that suffices for a specific loading through limit-state analysis. Specifically, [1] confirmed the results of [4] about the dimensionless minimum thickness of semicircular arches that equals $\mathrm{t} / \mathrm{R}=0.1075$ (t: thickness, $\mathrm{R}$ : radius) utilising radial cuts and $\mathrm{t} / \mathrm{R}=0.1095$ by implementing vertical cuts when subjected to self-weight. The above was achieved by the calculation of the limit thrust line associated with a five-hinge mechanism (a four-hinge mechanism would not be possible due to symmetry) as shown in Fig. 2a. Subsequently, [7] determined the minimum thickness of circular arches with different embrace angles $(\beta)$ subjected to their self-weight combined with inertial loading. The dimensionless minimum thickness was found through a variational formulation and the application of the principal of potential energy that allowed the evaluation of the minimum horizontal acceleration levels that turn the arch into a four-hinge mechanism (Fig. 2b). Furthermore, [22] estimated the minimum dimensionless thickness of pointed/gothic arches under self-weight, on the verge of becoming a five-hinge mechanism, via the thrust-line method. After Hooke [2], the catenary shape symbolised the perfect arch shape as it represented the optimal thrust line. [24] Highlighted the deviation of the resulting thrust line from the catenary centerline of a catenary arch, although he admitted that there is no minimum thickness for a two-dimensional catenary arch under self-weight. The justification is that, as the thickness of a catenary arch tends to zero (one-dimensional arch), the thrust line tends to follow a purely catenary shape and hence, it will always be confined within the arch's geometry (intrados and extrados). The above indicates that two-dimensional arches of catenary geometry, which are subjected only to their self-weigh, may constitute the best option among all the other arches of known geometries. Consequently, this work is based on [28] and aims to a apply limit-state analysis to parabolic arches, since they bear great resemblance and also are more commonly used than catenary arches, for both self-weight and inertial loads. Since there is a need to provide useful observations and solid knowledge about the strength and stability of "extraterrestrial" arches serving as shielding mechanisms for valuable assets on the Moon and Mars $[9,12]$, the research will be applied to low-gravity fields as well. These regolith-based arches are believed to function monolithically, regardless of the construction process (additive manufacturing, interlocking bricks, etc.). Furthermore, no material shear failure (including slipping at the base) is taken into consideration, so only the development of radial cuts is permissible everywhere along the arch geometry. The impact of stereotomy is outside the reach of this paper. 


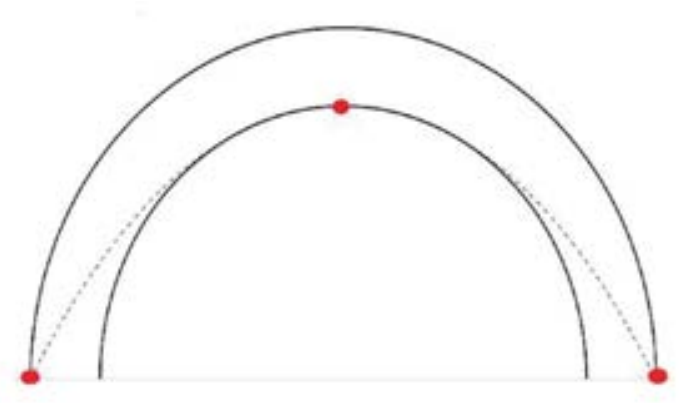

(a)

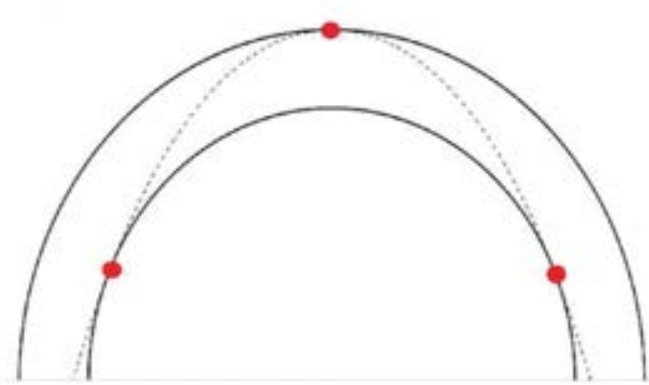

(b)

Figure 1: Statically determinate semicircular arch under its self-weight, with its (a) maximum and (b) minimum thrust line.

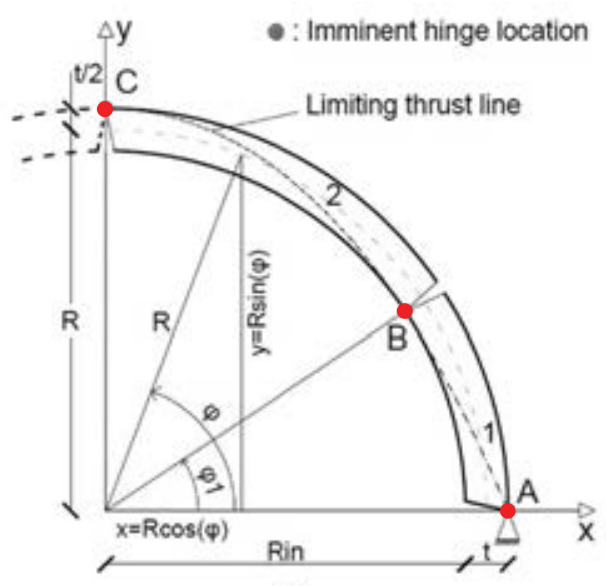

(a)
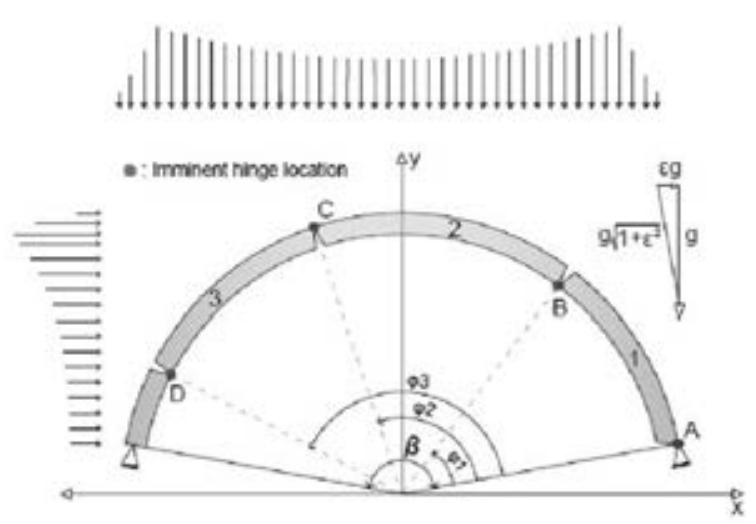

(b)

Figure 2: Statically determinate semicircular arch under its self-weight, with its (a) maximum and (b) minimum thrust line.

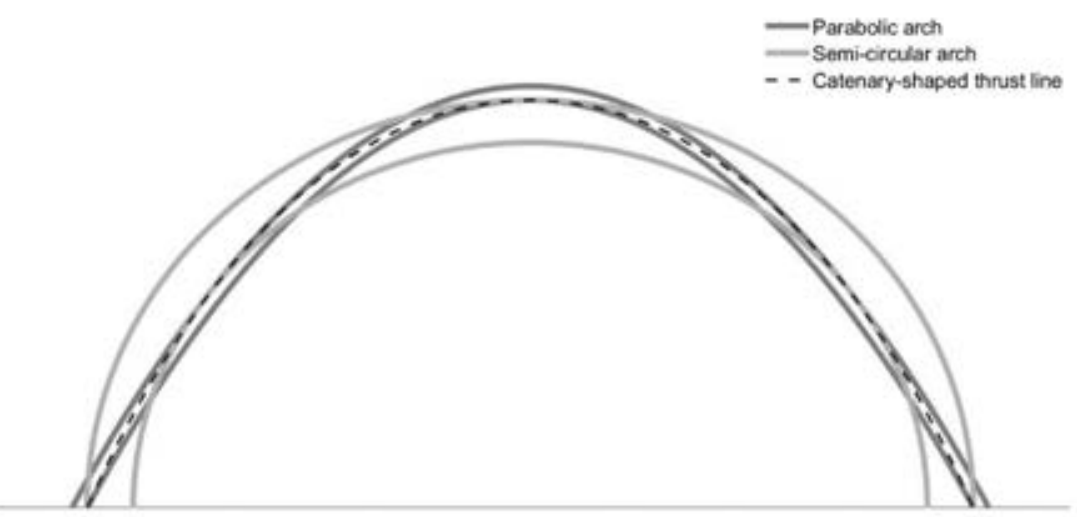

Figure 3: Qualitative illustration presenting the distinctions between the symmetric five hinge mechanism of a semi-circular and a parabolic arch under self-weight. The thrust line is represented by a catenary (black dashed line) and the two optimal arches with continues grey-scaled lines.

This study also aims to contrast the findings of the parabolic arch with those of circular arches, which are the most typical. Within Figure 3 lies the source of motivation for the qualitative identification of the failure mechanism for parabolic arches. Under self-weight only, the same thrust line (black dotted line) is marginally fitted inside both a semicircular and a parabolic (grey-scaled lines) arch with a catenary form (that bears a great resemblance to the actual thrust 
lines, even though it is not an admissible one). Clearly, there are two major points to be taken from Figure 3: a) The parabolic arch's five-hinge mechanism is essentially opposite than that of the semicircular, and b) the parabolic arch is substantially thinner than the semicircular. The inreference structure is a parabolic arch with an aspect ratio of $c=1 / 2$. (as shown in Fig. 4a). Since it is closely analogous to semicircular arches, this aspect ratio is of special importance.

\section{ANALYTICAL FORMULATION}

The analytical formulation of the symmetric five-hinge mechanism owing to self-weight for the parabolic arch is presented (see Fig. 4a). One may find details about the analytical procedure and the derivation of the pertinent equations in [28] however, selective parts of the analytical procedure are presented herein for reasons of better understanding.

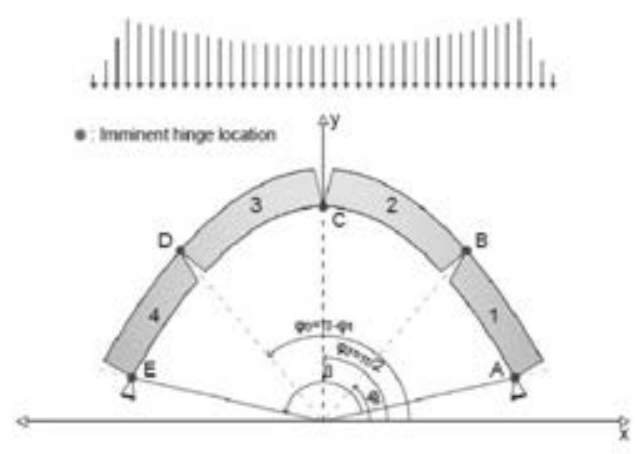

(a)

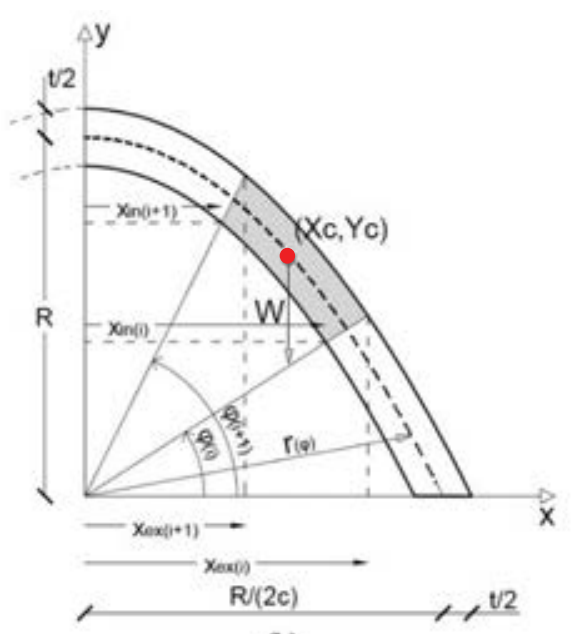

(b)

Figure 4: Parabolic arch: (a) symmetric five-hinge mechanism of the arch with $c=1 / 2$ and embrace angle $\beta$, when subjected to its self-weight and (b) reference system used in this study.

\subsection{Equations of the parabolic arch}

The generic equation that describes a parabola is:

$$
y=\alpha-b x^{2}
$$

The Cartesian coordinates of a parabolic arch can be represented using the following equation, assuming $c$ : rise over span ratio (this is true only for an embrace angle of $180^{\circ}$ ) and R: rise of a parabolic arch.

$$
y=R-\frac{4 c^{2}}{R} x^{2}
$$

Finally, after applying simple calculus, the Cartesian coordinates have been converted to polar and the geometry of the parabolic arch (see Fig. 4b) is expressed as follows:

$$
\left\{\begin{array}{lr}
r_{\left(\varphi_{i}\right)}=\left\{\frac{-\sin \left(\varphi_{i}\right)+\sqrt{\sin ^{2}\left(\varphi_{i}\right)+16 c^{2} \cos ^{2}\left(\varphi_{i}\right)}}{8 c^{2} \cos ^{2}\left(\varphi_{i}\right)}\right\} R & \forall \varphi_{i} \in\left[0, \frac{\pi}{2}\right) \cup\left(\frac{\pi}{2}, \pi\right] \\
r_{\left(\varphi_{i}\right)}=R & \text { for } \quad \varphi_{i}=\frac{\pi}{2}
\end{array}\right.
$$




\subsection{Moment equilibrium at the onset of the five-hinge mechanism}

The imminent hinges (at a state of equilibrium) of the collapse mechanism of the parabolic arch will appear at the intrados, where they would appear at the extrados for a semicircular arch and vice versa (Figs. 2a and 4a). The symmetric right part of the collapse mechanism is depicted in Figure 5. The gravitational multiplier, $\alpha=\frac{g^{\prime}}{g}$, is defined as the ratio of the gravitational acceleration on other planetary surfaces, $g^{\prime}$, over the terrestrial acceleration, $g=9.81 \frac{\mathrm{m}}{\mathrm{s}^{2}}$, in order to describe different gravitational environments. Additionally, the factor, $\mathrm{L}=\frac{m_{\text {total }}}{m_{\text {inertial }}}$ is measured as the ratio of the structure's total mass and the additional infill mass $\left(m_{\text {total }}=\right.$ $\left.m_{\text {structure }}+m_{\text {infill }}\right)$, over the mass that corresponds to the inertial loading $\left(m_{\text {inertial }}\right)$ which is a ratio of the $m_{\text {total }}$. For terrestrial applications where $\alpha=1$, it is assumed that there is no extra infill and thus, the total mass fully participates to the inertial loads $\left(m_{\text {total }}=\right.$ $\left.m_{\text {structure }}=m_{\text {inertial }}\right)$ and $L=1$.

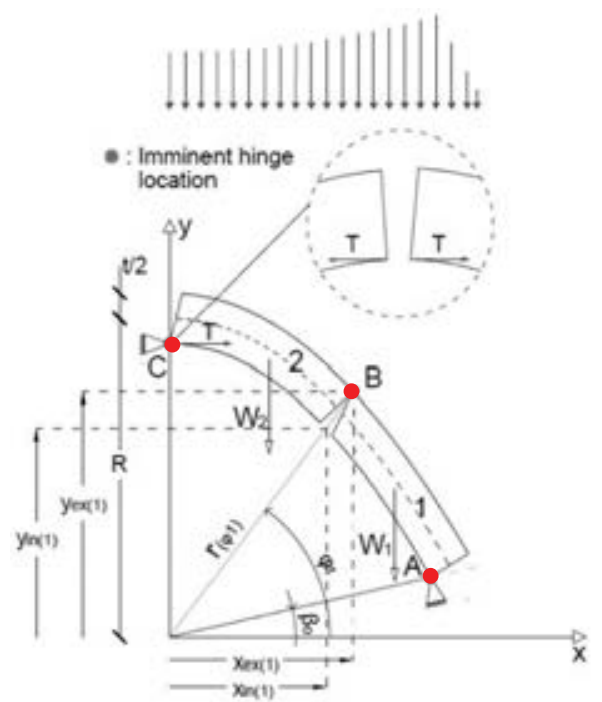

Figure 5: System of reference of the right symmetric part of the collapse mechanism of a parabolic arch subjected only to its self-weight.

Regarding the half right part (Fig. 5) of the arch, after applying a moment equilibrium about the point $\mathrm{A}$, the minimum horizontal thrust at the intrados of the crown, $T$, is calculated. Considering the low gravity effect $(\alpha)$ and the additional vertical (shielding) loading $(\mathrm{L})$, the weight of the segment (1-2) is taken equal to $\alpha \mathrm{LW}_{12}$. The moment equilibrium is represented by the following equation:

$$
\begin{gathered}
\text { Segment }(1-2): \mathrm{T}\left(\left\{\mathrm{R}-\frac{\mathrm{t}}{2}\right\}-\sin \left(\beta_{0}\right)\left\{\mathrm{r}_{0}-\frac{\mathrm{t}}{2}\right\}\right)-\alpha \mathrm{LW}_{12}\left(\cos \left(\beta_{0}\right)\left\{\mathrm{r}_{0}-\frac{\mathrm{t}}{2}\right\}-\mathrm{x}_{\mathrm{c} 12}\right)=0 \\
\Rightarrow \mathrm{T}=\frac{\alpha \mathrm{LW}_{12}\left(\cos \left(\beta_{0}\right)\left\{\mathrm{r}_{0}-\frac{\mathrm{t}}{2}\right\}-\mathrm{x}_{\mathrm{c} 12}\right)}{\left\{\mathrm{R}-\frac{\mathrm{t}}{2}\right\}-\sin \left(\beta_{0}\right)\left\{\mathrm{r}_{0}-\frac{\mathrm{t}}{2}\right\}} \quad, \quad \beta_{0} \neq \frac{\pi}{2}
\end{gathered}
$$

where: $\mathrm{W}_{12}$ represents the weight of half the arch

$\mathrm{x}_{\mathrm{c} 12}$ is the abscissa of the center of gravity of the half arch

$\mathrm{r}_{0}$ is the radius at an angle $\beta_{0}$ 
After assuming a radial cut as seen in Fig. 5, moment equilibrium of the arch's upper portion (segment 2) over hinge B gives,

Segment(2):

$$
\begin{gathered}
-\alpha \mathrm{LW}_{2}\left(\cos \left(\varphi_{1}\right)\left\{\mathrm{r}_{\left(\varphi_{1}\right)}+\frac{\mathrm{t}}{2}\right\}-\mathrm{x}_{\mathrm{c} 2}\right)+\mathrm{T}\left(\sin \left(\varphi_{2}\right)\left\{\mathrm{r}_{\left(\varphi_{2}\right)}-\frac{\mathrm{t}}{2}\right\}-\sin \left(\varphi_{1}\right)\left\{\mathrm{r}_{\left(\varphi_{1}\right)}+\frac{\mathrm{t}}{2}\right\}\right)=0 \\
\Rightarrow-\alpha \mathrm{LW}_{2}\left(\cos \left(\varphi_{1}\right)\left\{\mathrm{r}_{\left(\varphi_{1}\right)}+\frac{\mathrm{t}}{2}\right\}-\mathrm{x}_{\mathrm{c} 2}\right) \\
+\frac{\alpha \mathrm{LW}_{12}\left(\cos \left(\beta_{0}\right)\left\{\mathrm{r}_{0}-\frac{\mathrm{t}}{2}\right\}-\mathrm{x}_{\mathrm{c} 12}\right)}{\left\{\mathrm{R}-\frac{\mathrm{t}}{2}\right\}-\sin \left(\beta_{0}\right)\left\{\mathrm{r}_{0}-\frac{\mathrm{t}}{2}\right\}}\left(\left\{\mathrm{R}-\frac{\mathrm{t}}{2}\right\}-\sin \left(\varphi_{1}\right)\left\{\mathrm{r}_{\left(\varphi_{1}\right)}+\frac{\mathrm{t}}{2}\right\}\right)
\end{gathered}
$$

where $\mathrm{W}_{2}$ is the weight of the segment 2

$\mathrm{x}_{\mathrm{c} 2}$ is the abscissa of the center of gravity of the segment 2

$\mathrm{r}_{\left(\varphi_{1}\right)}$ represents the radius at an angle $\varphi_{1}$

As Eq. 5 is solved with respect to $t$ (arch thickness), a transcendental equation is obtained that links the unknown rupture angle $\varphi_{1}$ with the unknown minimum thickness $t$. The following is the general representation of this equation:

$$
t=f\left(\varphi_{1}\right)
$$

If adopt the horizontal axis $x(y=0)$ as a level of reference, the potential energy of the parabolic arch following an embrace angle $\beta$ (Fig. 7b) will be,

$$
V=W_{\text {arch }} \cdot y_{0}
$$

where $\mathrm{W}_{\mathrm{arch}}$ represents the weight of the parabolic arch and $y_{0}$ is the ordinate of the center of gravity of the parabolic arch. By substituting Eq. 6 in Eq. 7, we have,

$$
V\left(\varphi_{1}\right)=W_{\text {arch }} \cdot y_{0}
$$

Based on the theoryme of stationary potential energy, the geometrically admissible hinge mechanism (Fig. 4a) is in a state of equilibrium if and only if the total potential energy is stationary, i.e.,

$$
\delta V\left(\varphi_{1}\right)=0 \Rightarrow \frac{d V\left(\varphi_{1}\right)}{d \varphi_{1}} \delta \varphi_{1}=0 \Rightarrow \frac{d V\left(\varphi_{1}\right)}{d \varphi_{1}}=0
$$

After solving the system of Eqs. (6) and (9) one may evaluate the unknown location of the rupture angle $\varphi_{1}$ along with the minimum width $t$ of a parabolic arch for a given embrace angle $\beta$.

\subsection{Results - considering self-weight for terrestrial gravity}

Figure 6 contrasts the limit thrust lines regarding semicircular and parabolic arches with embrace angle $\beta=180^{\circ}$ subjected to their self-weight. As it is expected, the collapse mechanisms are exactly opposite. Specifically, in the case of the semi-circular arch, the thrust line starts at the extrados of the base, it touches the intrados at $35.52^{\circ}$ and then the extrados at the 
crown, while for the parabolic arches the limit thrust line initiates at the intrados at the base, then touches the extrados at $43.81^{\circ}$ and the intrados at the crown. Most importantly, the minimum thickness of parabolic arches is $\frac{t}{R}=0.0239$, while the corresponding minimum thickness for semicircular arches is $\frac{t}{R}=0.1075$.

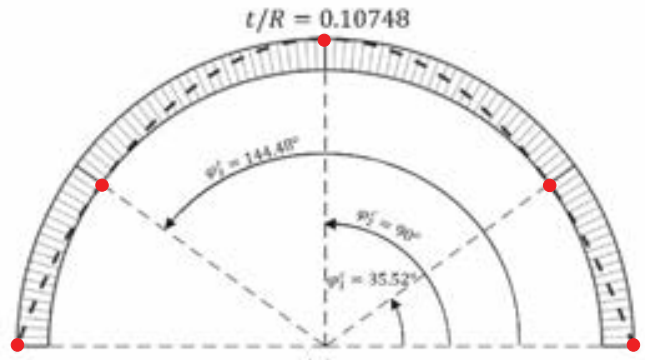

(a)

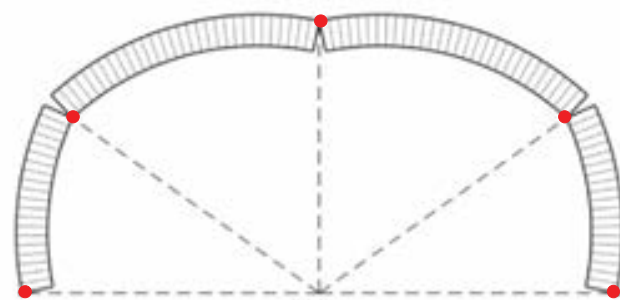

(c)

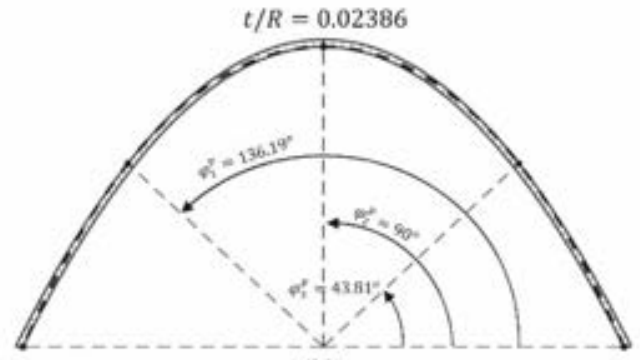

(b)

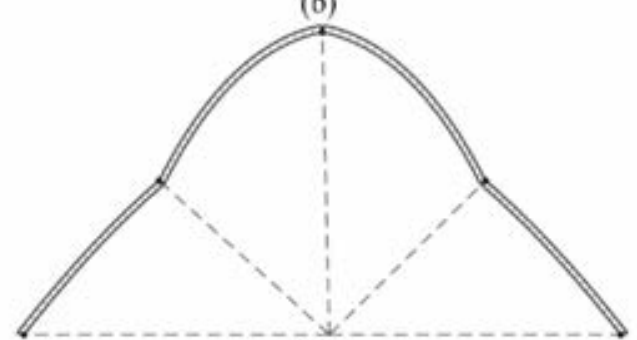

(d)

Figure 6: Limit thrust line for (a) semicircular and (b) parabolic arches; symmetric five-hinge mechanism of (c) semicircular and (d) parabolic arches when subjected to their self-weight.

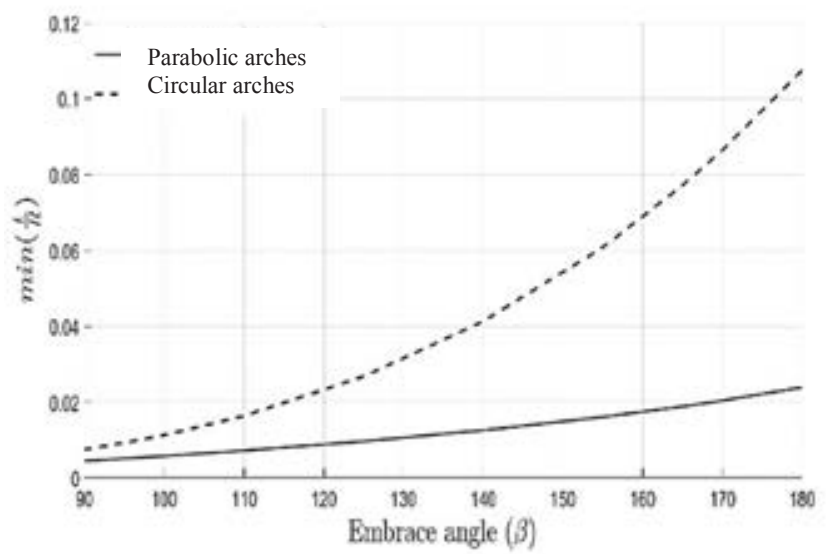

Figure 7: Minimum t/R and versus various embrace angles $(\beta)$ for circular (solid line) and parabolic arches (dashed line) subjected only to their self-weight.

Also, figure 7 compares the minimum dimensionless thickness of circular to parabolic arches for various embrace angles, $\beta$. Evidently, the parabolic arches (with $c=\frac{1}{2}$ ), always require thinner geometries to support their self-weight than their circular counterparts, regardless of $\beta$. Moreover, the difference between the $\min \left(\frac{t}{R}\right)$ of a circular and parabolic arch decreases for smaller values of $\beta$ (Fig. 7).

\section{IDENTIFICATION OF THE FOUR-HINGE MECHANISM}

When an arch is subjected to horizontal acceleration $(\varepsilon)$, it exhibits a four-hinge non-symmetric mechanism $[7,28]$. As in the case of the five-hinge mechanism, the failure mechanisms 
are exactly opposite. To facilitate comparisons between the different failure mechanisms of the two arch geometries examined in this work, the following assumptions regarding the rupture angles have been made. In the case of circular geometry, $\varphi_{1}=\varphi_{1}^{C}, \varphi_{2}=\varphi_{2}^{C}$ and $\varphi_{3}=\varphi_{3}^{C}$ while for the parabolic geometry, $\varphi_{1}=\pi-\varphi_{1}^{P}, \varphi_{2}=\pi-\varphi_{2}^{P}$ and $\varphi_{3}=\pi-\varphi_{3}^{P}$.

\subsection{Moment equilibrium at the onset of the four-hinge mechanism}

Following similar procedure with that of the five-hinge mechanism and after implementing moment equilibrium, one may end up to the following equation that links the lateral acceleration level $(\varepsilon)$ with the three rapture angles $\left(\varphi_{1}, \varphi_{2}, \varphi_{3}\right)$ and the minimum thickness $(t)$ [28].

$$
\varepsilon=f\left(\varphi_{1}, \varphi_{2}, \varphi_{3}, t\right)
$$

To calculate the rupture angles, $\varphi_{1}, \varphi_{2}, \varphi_{3}$ referring to a minimum $t / R$ and a given horizontal acceleration level, $\varepsilon$, the principle of potential energy is applied. Thus, the total energy of the system is expressed as the work of all forces acting on the structure with reference $y=0$. The loading on a parabolic arch subjected to a horizontal acceleration, $\varepsilon g$, is equivalent to the gravity loading on the same parabolic arch when it is rotated by an angle, $\omega=\tan ^{-1} \frac{\varepsilon}{\alpha}$ from the vertical under a new gravitational field, $\hat{g}=g \sqrt{\alpha^{2}+\varepsilon^{2}}$. The resultant gravitational field is $\hat{g}$ and the potential energy can be expressed as

$$
\begin{gathered}
\hat{V}=\widehat{W} \cdot \hat{y}=\sqrt{\alpha^{2}+\varepsilon^{2}} W \hat{y} \quad \neq V \quad \text { or } \\
V\left(\varphi_{1}, \varphi_{2}, \varphi_{3}, \varepsilon\right)=\sqrt{1+f\left(\varphi_{1}, \varphi_{2}, \varphi_{3}, t\right)^{2}} W \hat{y}
\end{gathered}
$$

According to potential energy principle, the four-hinge mechanism is in equilibrium if, and only if, the variational work increment vanishes for arbitrary geometrically admissible variations. Therefore, the potential energy of the system needs to be stationary, $\delta V=0$. The potential energy is stationary when

This leads to the following expressions:

$$
\delta V=V\left(\varphi_{i} \pm \delta \varphi_{i}\right)-V\left(\varphi_{i}\right)=\frac{\partial V\left(\varphi_{i}, \varepsilon\right)}{\partial \varphi_{i}} \delta \varphi_{i}=0 \Rightarrow \frac{\partial V\left(\varphi_{i}, \varepsilon\right)}{\partial \varphi_{i}}=0
$$

$$
\begin{aligned}
& \frac{\partial V\left(\varphi_{1}, \varphi_{2}, \varphi_{3}, \varepsilon\right)}{\partial \varphi_{1}}=0 \\
& \frac{\partial V\left(\varphi_{1}, \varphi_{2}, \varphi_{3}, \varepsilon\right)}{\partial \varphi_{2}}=0 \\
& \frac{\partial V\left(\varphi_{1}, \varphi_{2}, \varphi_{3}, \varepsilon\right)}{\partial \varphi_{3}}=0
\end{aligned}
$$

Where by substituting Eq. 11 to Eqs. 12, 13, 14

$$
\frac{\partial V\left(\varphi_{1}, \varphi_{2}, \varphi_{3}, \varepsilon\right)}{\partial \varphi_{i}}=\frac{\partial f\left(\varphi_{1}, \varphi_{2}, \varphi_{3}, t\right)}{\partial \varphi_{i}} W \hat{y} \frac{f\left(\varphi_{1}, \varphi_{2}, \varphi_{3}, t\right)}{\sqrt{1+f\left(\varphi_{1}, \varphi_{2}, \varphi_{3}, t\right)^{2}}}=0 \quad i \in[1,2,3]
$$


Since the horizontal acceleration is not zero, $\varepsilon=f\left(\varphi_{1}, \varphi_{2}, \varphi_{3}, t\right) \neq 0$, and that $\hat{y}$ is nonzero as well, equation (15) yields

$$
\frac{\partial f\left(\varphi_{1}, \varphi_{2}, \varphi_{3}, t\right)}{\partial \varphi_{i}}=\frac{\partial \varepsilon}{\partial \varphi_{i}}=0
$$

Equation (10) combined with Eq. (16) for $i \in[1,2,3]$ yield to a $4 \times 4$ system of equations that can be solved in order to compute the four unknowns $\varphi_{1}, \varphi_{2}, \varphi_{3}$ and $t$ for a certain acceleration level and embrace angle $\beta$.

\subsection{Results - considering self-weight and inertial acceleration for terrestrial gravity}

The minimum dimensionless thickness of the parabolic and circular arches are presented in figure 8 for terrestrial gravitational conditions $(\alpha=1)$ and assuming that the whole mass contributes to inertial forces $(L=1)$. As anticipated, Fig. 8 hilights i) the need for thicker geometries as the lateral inertial load increases and ii) the need for thinner geometries as the embrace angle decreases, for both the circular and parabolic case. In spite of the self-weight consideration where the parabolic arches have been proved more optimal than the corresponding circular ones, figure 8 indicates that after a certain value of embrace angle $(\beta)$ and lateral inertial load $(\varepsilon g)$, the circular arches tend to be more efficient.

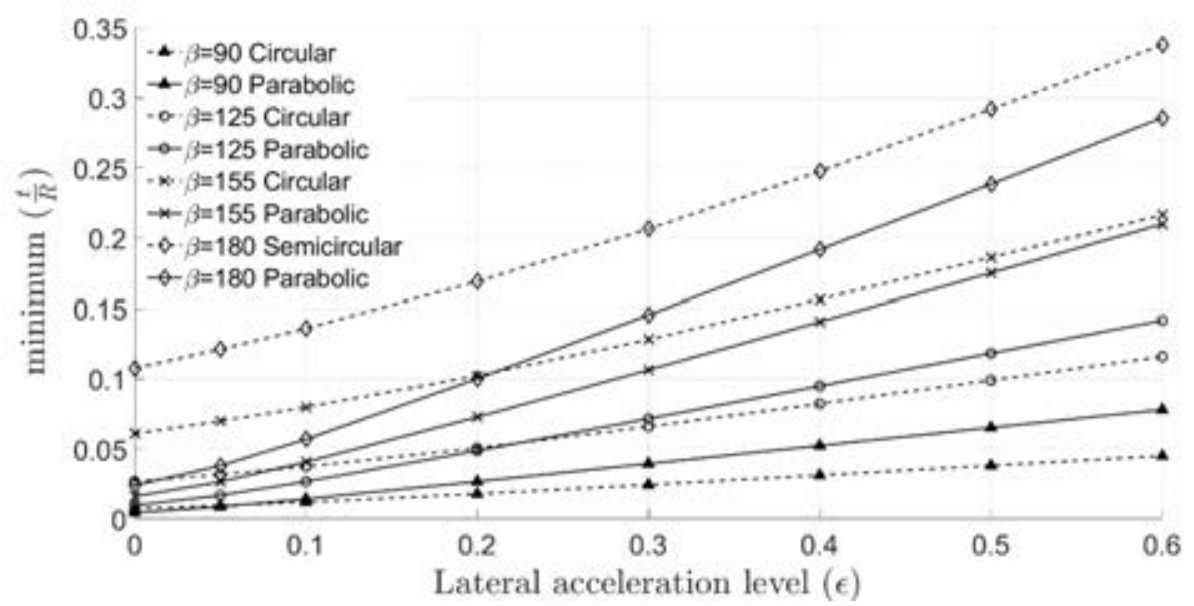

Figure 8: Minimum $\frac{t}{R}$ versus horizontal acceleration for circular and parabolic arches with embrace angles

$$
\left\{\beta=90^{\circ}, 125^{\circ}, 155^{\circ} \text { and } 180^{\circ}\right\} .
$$

Figure 9 shows the different limit thrust lines for semicircular and parabolic arches under terrestrial gravity $\left(g=9.81 \frac{\mathrm{m}}{\mathrm{s}^{2}}\right)$ and horizontal accelerations, $\varepsilon g=\{0.1 ; 0,2 ; 0.4 ; 0.6\} g$ together with the corresponding rupture angles. Regarding the parabolic arches, given a horizontal acceleration directed from left to right, the failure mechanism always starts from the intrados at the left springing, then there is $\varphi_{1}$ at the extrados, $\varphi_{2}$ at the intrados and $\varphi_{3}$ at the extrados of the right springing. 

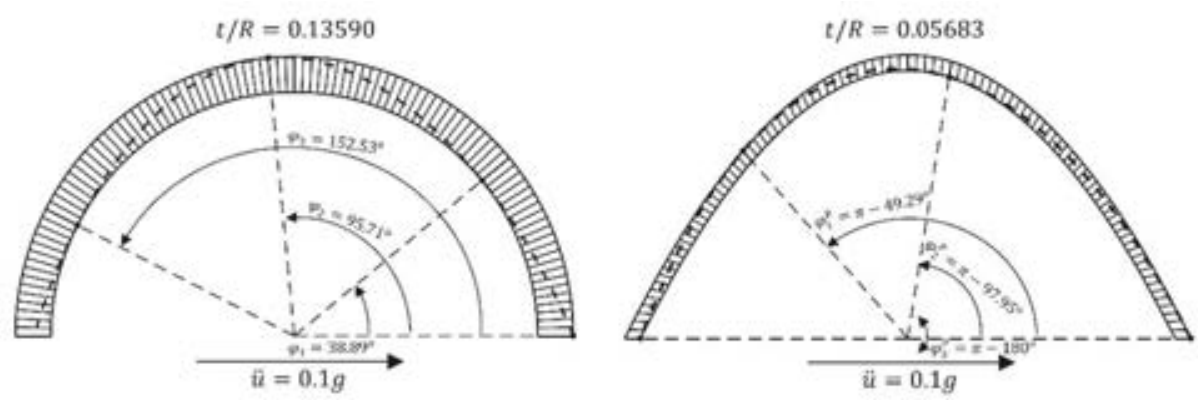

(a)
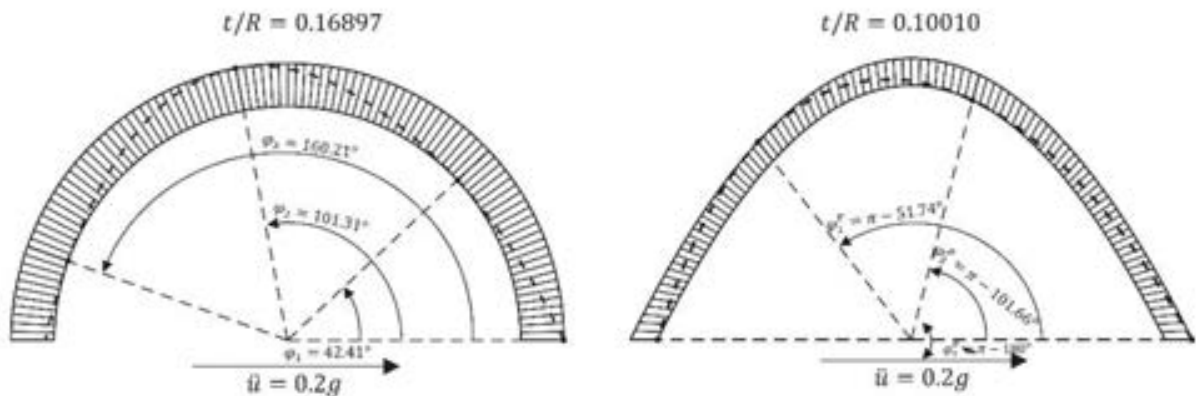

(b)
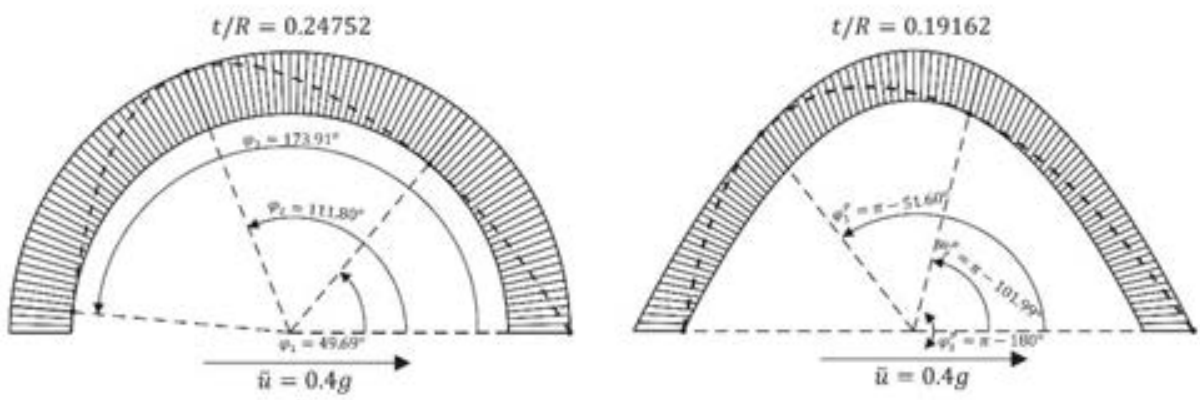

(c)
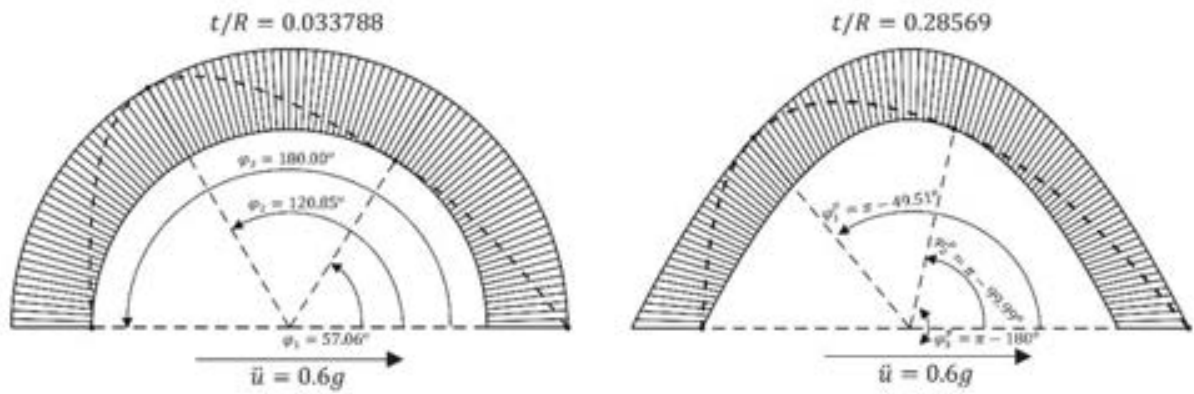

(d)

Figure 9: Contrast of the limit thrust lines for semicircular and parabolic arches under their self-weight and horizontal acceleration (from left to right) of (a) $\varepsilon g=0.1 g$; (b) $\varepsilon g=0.2 g$; (c) $\varepsilon g=0.4 g$ and (d) $\varepsilon g=0.6 g$.

\section{ARCHES IN LOW-GRAVITY CONDITIONS}

The effects of low gravity on the stability of parabolic and circular arches are discussed in this section. The gravitational multiplier, $\alpha=\frac{g^{\prime}}{g}$, is used to account for the influence of low gravity, with values of 0.38 and 0.17 with respect to the martian and lunar gravitational fields. Given that these arches might be used as possible shielding structures against radiation and intense temperature fluctuations, loose regolith material at the top could be needed to achieve a safe thickness of about $250 \mathrm{~cm}$ [27]. 
$L=\frac{m_{\text {total }}}{m_{\text {inertia }}}=\frac{m_{\text {structure }}+m_{\text {infill }}}{m_{\text {inertia }}}$, in this case the factor $L$ takes the additional mass due loose regolith infill into consideration $\left(m_{\text {infill }} \neq 0\right)$ and since not all of it contributes to inertial loading, $L$ may take the values higher than 1 . Specifically, $L=1,2,3,4$ is considered herewith, where $L=1$ signifies that the entire mass contributes to inertia, $m_{\text {total }}=m_{\text {inertia }}$. Figure 10 roughly depicts the loose regolith infill atop a parabolic arch. As the structure is exposed to more regular, low-impact ground motions, (Fig. 10a), the infill remains intact (similarly to Serviceability Limit State, SLS). Thus, in this case $L=1$ since the infill participates entirely to the inertial loading. However, when the structure is exposed to more intense ground motions, it is highly possible that the infill will yield and then partially collapse, as it is shown in figure $10 \mathrm{~b}$ (similarly to the Ultimate Limit State, ULS). In this case, the infill contributes only partially to the inertia of the structure, as it is not monolithically attached to it and therefore $L>1$. The intensity of ground motion, the geometry of the arch, how the loose material has been connected to the arch, the coherence between the loose and the structural material, the gravitational conditions, among other considerations, all play a significant role in this partial collapse, and further analysis is required before designing such structures.
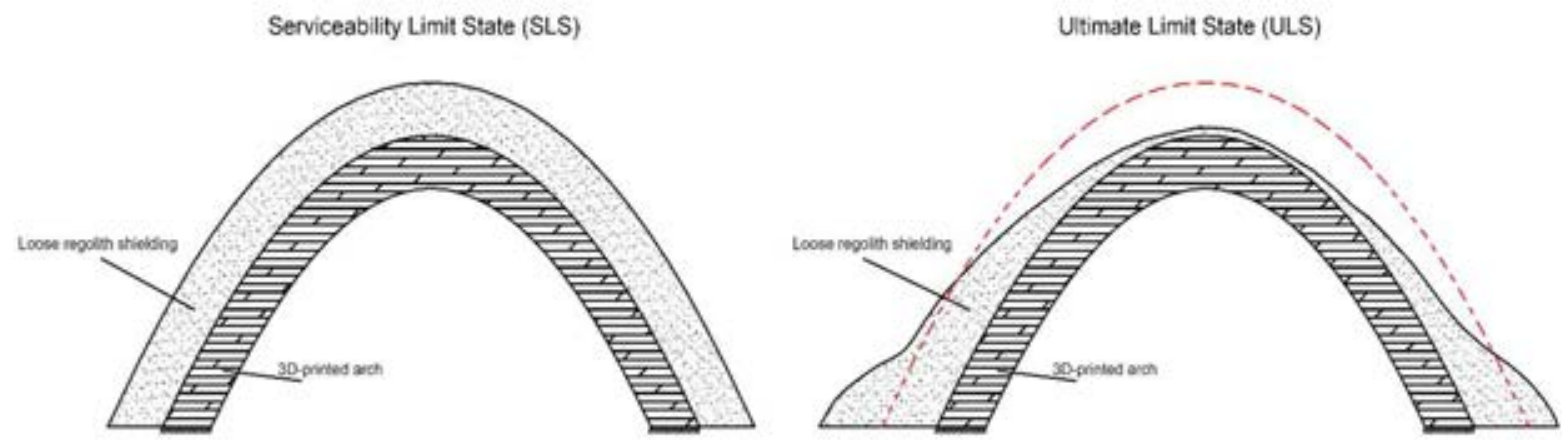

Figure 10: Minimum $\frac{t}{R}$ versus horizontal acceleration for circular and parabolic arches with embrace angles $\left\{\beta=90^{\circ}, 125^{\circ}, 155^{\circ}\right.$ and $\left.180^{\circ}\right\}$.

Intriguingly, for both parabolic and circular arches, the phenomenon develops a self-similar behaviour portrayed by the master curves of the minimum $\frac{t}{R}$ and rupture angles versus the dimensionless horizontal acceleration, $\frac{\varepsilon}{\alpha L}$ in figures 11. In particular, each pair of $\beta$ and minimum $\frac{t}{R}$ corresponds to a unique vector of $\left\{\frac{\varepsilon}{\alpha L}, \varphi_{1}, \varphi_{2}, \varphi_{3}\right\}$. After observing figure 11 one may notice that for high values of $\frac{\varepsilon}{\alpha L}$ (which translates to either substantial inertial loads or low-gravity conditions and no additional vertical loading) the parabolic arch is always thinner than the circular arch for $\beta=180^{\circ}$. Nevertheless, for smaller embrace angles, there is a certain value of $\frac{\varepsilon}{\alpha L}$ after which the circular arch becomes more efficient (in terms of material usage) than the parabolic. A more thorough investigation of the self-similar behavior phenomenon mentioned above is presented in [28]. 


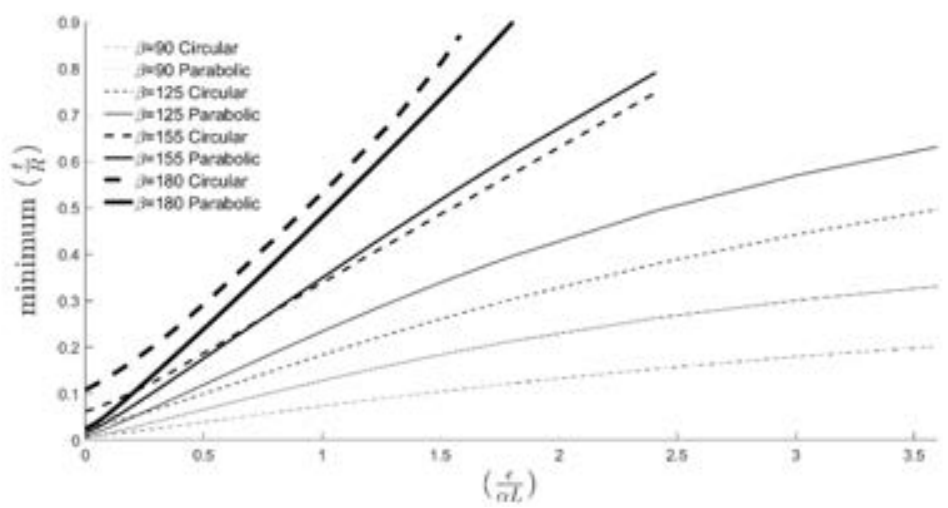

Figure 11: Master curves of the minimum required $\frac{t}{R}$ versus the dimensionless inertial loading, $\frac{\varepsilon}{\alpha L}$, for both circular and parabolic arches.

\section{CONCLUSIONS}

This work is based on that of [28] and has investigated via limit-state analysis the structural behavior of circular and parabolic arches when they are subjected to self-weight, additional vertical weight and inertial loading in low-gravity conditions. More specifically, this research uses a variational formulation to evaluate the minimum necessary thickness of the various arches in order for them to avoid becoming a mechanism under various load combinations and gravitational fields. The findings indicate that contrary to circular arches, parabolic arches need a far lower minimum thickness to withstand their own weight and display the symmetrically opposite five-hinge mechanism. This manifests the "geometrical superiority" among structures that resemble the catenary shape (parabolic) under self-weight. It is observed that larger inertial loading results in higher minimum thickness, smaller embrace angles lead to thinner arches.

Interestingly, both the symmetrical five hinge mechanism formed due to the effect of selfweigh and the asymmetrical four-hinge mechanism developed due to the horizontal loading are opposite for the two types of arches. Nevertheless, regarding the four-hinge mechanism, the mechanism pattern shows a significant similarity between these two arch geometries, in the sense that as the inertial loading increases, in all cases, the mechanism tends to move "upstream" against the horizontal load in order for the arches to accommodate their limit thrust lines.

Moreover, a self-similar behaviour has been observed, revealing that every arch of a certain geometry type and embrace angle $(\beta)$ that is subjected to a "universal" dimensionless inertial load $\frac{\varepsilon}{\alpha L}$, always results in the same minimum thickness and rupture angles $\left\{\frac{t}{R}, \varphi_{1}, \varphi_{2}\right.$, $\left.\varphi_{3}\right\}$. One may refer to [28] for further information about the analytical procedure, the justification of the self-similarity and the conditions under which the parabolic geometry is more efficient that the corresponding circular and vise versa.

Given all the above, the need to optimise the arch geometry becomes apparent in lowgravity conditions, as shown in Section 5, since the required minimum thickness can become prohibitively large.

\section{REFERENCES}

[1] N. Makris and H. Alexakis. The effect of stereotomy on the shape of the thrust-line and the minimum thickness of semicircular masonry arches. Archive of Applied Mechanics, 83(10),1511-1533, 2013. 
[2] R. Hooke. A description of helioscopes, and some other instruments. London, 1675.

[3] Couplet P. De la pouss'ee des voûtes. Histoire de l'Acad'emie Royale des Sciences, Paris (1731, 1732), pp. 79-117 and pp. 117-141 (1729, 1730).

[4] M. Milankovitch. Beitrag zur Theorie der Druckkurven. Dissertation zur Erlangung der Doktorwürde, K.K. technische Hochschule, Vienna, 1904.

[5] P. Clemente. Introduction to dynamics of stone arches. Earthquake Engineering and Structural Dynnamics, 27,513-522, 1998.

[6] J. Ochsendorf. Collapse of masonry structures. PhD Dissertation. Cambridge: Department of Engineering, University of Cambridge, 2002.

[7] H. Alexakis, N. Makris. Limit equilibrium analysis and the minimum thickness of circular masonry arches to withstand lateral inertial loading. Archive of Applied Mechanics, 84(5),757-772, 2014.

[8] M. Trial. Some effects of declining US university civil engineering enrollments on the space enterprise. ASCE, $7^{\text {th }}$ International Conference and Exposition on Engineering, Construction, Operations, and Business in Space (Space 2000), Albuquerque, New Mexico, United States, February 27-March 2, 2000.

[9] N. Kalapodis, G. Kampas, O-J. Ktenidou. A review towards the design of extraterrestrial structures: from regolith to human outposts. Acta Astronautica, 175, 540-569, 2020.

[10] AM. Jablonski, KA. Ogden. Technical requirements for lunar structures. Journal of Aerospace Engineering, 21(2),72-90, 2008.

[11] G. Reitz, T. Berger, D. Matthiae. Radiation exposure in the moon environment. Planetary and Space Science,74(1),78-83, 2012.

[12] S. Wilkinson, J. Musil, J. Dierckx, I. Gallou, X. De Kestelier. Autonomous Additive Construction on Mars. Earth and space 2016: engineering for extreme environments - proceedings of the 15th biennial international conference on engineering, science, construction and operations in challenging environments, p.343-53, 2016.

[13] D. Rapp. Use of extraterrestrial resources for human space missions to moon or mars; 2018.

[14] BJ. Chow, T. Chen, Y. Zhong, Y. Qiao. Direct formation of structural components using a Martian soil simulant. Scientific Reports,7(1), 1151, 2017.

[15] A. Goulas, JGP. Binner, RA. Harris, RJ. Friel. Assessing extraterrestrial regolith material simulants for in-situ resource utilisation based 3D printing. Applied Materials Today, 6,54-61, 2017.

[16] Y. Nakamura, GV. Latham, HJ. Dorman. How we processed Apollo Lunar seismic data. Physics of the Earth and Planetary Interiors, 21(2-3), 218-224, 1980.

[17] F. Duennebier, J. Dorman, D. Lammlein, G. Latham, Y. Nakamura. Meteoroid flux from passive seismic experiment data. In: Proceedings. lunar science conference, $6^{\text {th }}$, p. $2417-$ 2426, 1975.

[18] N. Kalapodis, G. Kampas, O-J. Ktenidou. Revisiting the fundamental structural dynamic systems: the effect of low gravity. Archive of Applied Mechanics, 10, 1861-1884, 2019. 
[19] N. Kalapodis, G. Kampas, J. Webb, O-J. Ktenidou. The effect of self-weight on freestanding blocks. In: 2nd International Conference on Natural Hazards \& Infrastructures (ICONHIC), 2019.

[20] E. Ricci, A. Fraddosio, MD. Piccioni, E. Sacco. A new numerical approach for determining optimal thrust curves of masonry arches. European Journal of Mechanics-A/Solids, 75, 426-442, 2019.

[21] H. Alexakis, N. Makris. Minimum thickness of elliptical masonry arches. Acta Mechanica, 224(12), 2977-2991, 2013.

[22] D. Nikolić. Thrust line analysis and the minimum thickness of pointed masonry arches. Acta Mechanica, 228(6),2219-2236, 2017.

[23] G. Tempesta, S. Galassi. Safety evaluation of masonry arches. A numerical procedure based on the thrust line closest to the geometrical axis. International Journal of Mechanical Science, 155, 206-221, 2019.

[24] D. Nikolić. Catenary arch of finite thickness as the optimal arch shape. Structural and Multidisciplinary Optimization, 60(5),1957-1966, 2019.

[25] P. Block, M. DeJong, J. Ochsendorf. As hangs the flexible line: equilibrium of masonry arches. Nexus Network Journal, 8(2),13-24, 2006.

[26] A. Iserles. A first course in the numerical analysis of differential equations. Cambridge University Press; 2008.

[27] H. Benaroya, L. Bernold, KM. Chua. Engineering, design and construction of lunar bases. Journal of Aerospace Engineering,15(2), 33-45, 2002.

[28] Kampas, G., Kalapodis, N., McLean, T., Málaga-Chuquitaype, C. Limit-state analysis of parabolic arches subjected to inertial loading in different gravitational fields using a variational formulation. Engineering Structures, 228, 111501, 2021. 